\title{
Spin-Orbit Effect on Thermal Properties of Half-Heusler Alloy FeVSb
}

\author{
Anugrah Azhar* \\ Physics Study Program, Faculty of Sciences and Technology, Syarif Hidayatullah \\ State Islamic University Jakarta, Tangerang Selatan 15412, Indonesia
}

\begin{abstract}
FeVSb, a family of Half-Heusler alloy, is categorized as an n-type semiconductor which has a high figure of merit that makes this material to be one of the promising candidates for a thermoelectric device. In $\mathrm{FeVSb}, \mathrm{Fe}$ and $\mathrm{V}$ atoms which have $d$-orbitals can be considered to have spin-orbit interaction that can affect the electronic structure and thermal properties of this material. In order to investigate how strong the spin-orbit interaction affect this material, we do the first-principles study by implementing the spin-orbit interaction to investigate the change of the band structure and thermal properties of FeVSb. Our results show that the spinorbit interaction affects the band structure of the material indicated by the energy splitting in the electronic structure which increase the Seebeck coefficient, electrical conductivity, and thermal conductivity but slightly reduce the figure of merit values of $\mathrm{FeVSb}$.
\end{abstract}

Keywords: Spin-orbit; First-principles; FeVSb; Thermal properties.

*Corresponding author: anugrahazhar@uinjkt.ac.id

Article history: Received 22 Maret 2021, Accepted 19 Mei 2021, Published June 2021.

http://dx.doi.org/10.12962/j24604682.v17i2.8973

2460-4682 (c)Departemen Fisika, FSAD-ITS

\section{INTRODUCTION}

The discovery of Heusler Alloy (HA) can be traced to the last of the 90th century when $\mathrm{F}$. Heusler found that $\mathrm{Cu}_{2} \mathrm{MnAl}$ become magnetic although $\mathrm{Cu}, \mathrm{Mn}$, and $\mathrm{Al}$ are not magnetic by itself [1]. HA, having a general formula of $\mathrm{X}_{2} \mathrm{YZ}$ for fullHeusler (FH) and XYZ for half-Heusler $(\mathrm{HH})$, consists of $X$ and $\mathrm{Y}$ atoms that are transition metal elements, while $\mathrm{Z}$ is the main group elements [2]. Fascinatingly, the magnetic moment of this material can be predicted by counting its valance electrons, called the Slater-Pauling rule $[2,3]$. To obtain the desired physical properties of HA, one can modify the HA by changing its compositions through chemical substitutions and structural motifs [4]. Because of high tuning capability, the exploration of HA has been extensively explored to be implemented in technology such as spintronics [5, 6], shapememory materials [7], superconductors [8], and thermoelectric (TE) devices [9].

$\mathrm{HH}$ as one of the families of HA has been broadly explored because of its high figure of merit $(\mathrm{ZT})$ value around $\mathrm{ZT} \approx 1$ $[9,10]$. A high ZT value can be obtained by decreasing the thermal conductivity caused by electron and phonon, therefore there are several experimental methods that can be used to obtain a high ZT value such as nano-sized sample [11, 12], the void filling method [13], and manipulate the atomic size with higher size atom $[14,15]$.

By using first-principles study, Sukumu et al. report that half-Heusler compounds CoMSb $(\mathrm{M}=\mathrm{Sc}, \mathrm{Ti}, \mathrm{V}, \mathrm{Cr}$, and $\mathrm{Mn})$ as nonmagnetic compounds have carrier concentration dependence of Seebeck coefficient $(S)$ which are in good agreement with experimental values [16]. The cobalt based Heusler al- loys have been vastly studied by many researchers and show the good in agreement with the experimental results, however the iron-based Heusler alloys have not studied as much as the cobalt-based Heusler alloys. In order to explore and to give the information about the electronic and thermal properties of the iron-based Heusler alloy, we therefore perform a theoretical study to investigate the thermal properties of $\mathrm{FeVSb}$ as one of the iron-based Heusler alloys family by performing the density-functional theory (DFT) calculation using QuantumEspresso package [17, 18], followed by BoltzTraP calculation to obtain the thermal properties (electronic part) of the system [19]. In this study, we only deal with the thermal properties contributed by the electron because the thermal properties contributed by phonon needs quite expensive calculation.

\section{METHOD}

To obtain the electronic properties of $\mathrm{FeVSb}$, we perform the DFT calculations by running Quantum-Espresso (QE) package $[17,18]$ under a parallel scheme by using Message Passing Interface (MPI) to reduce the computational time [20]. We use Perdew-Burke-Ernzerhof (PBE) exchangecorrelation with semi core electron states for system without spin-orbit (wo-SO) interaction, while to do calculation of system with spin-orbit (SO) interaction, we use the fullrelativistic pseudopotential of the same type from the Psilibrary [21]. The wave function and the charge density cut-off used in this calculation for both systems are 60 and 650 Rydberg, respectively. BoltzTraP calculation is then performed to obtain the thermal properties of FeVSb system [19].

$\mathrm{FeVSb}$ crystalizes in $\mathrm{Cl}_{\mathrm{b}}$ crystal structure with the lattice 


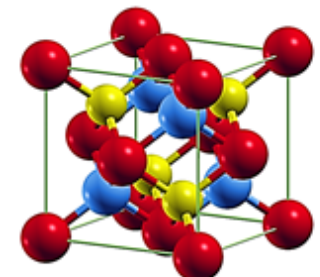

(a)

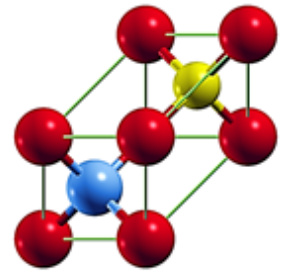

(b)
FIG. 1: (a) The conventional unit cell and (b) the primitive unit cell of $\mathrm{FeVSb}$. The Fe, $\mathrm{V}$, and $\mathrm{Sb}$ atoms are illustrated in red, blue, and yellow, respectively.

constant of $5.7758 \AA$. Fig. 1 is generated by Xcrysden to visualize the FeVSb crystal structure with Wyckoff positions of $\mathrm{Fe}$ (crimson red), $\mathrm{V}$ (metallic light blue), and $\mathrm{Sb}$ (metallic yellow) placed at $(0,0,0),(0.25,0.25,0.25)$, and $(0.75,0.75,0.75)$, respectively [2].

\section{RESULTS AND DISCUSSION}

\section{A. Band Structure}

The band structures of FeVSb for both systems are calculated through the self-consistent field (SCF) calculation with $101010 \mathrm{k}$-points grid mesh. In the non-self-consistent field (NSCF) calculation, we dense the k-points grid mesh into 606060 and use the tetrahedron method to obtain a very accurate chemical potential value. For the band structure calculation, we use $100 \mathrm{k}$-points along the k-paths for each band to acquire a very smooth band structure. The convergence threshold for SCF, NSCF, and BANDS calculations are set to be $10^{-6}$. The Fermi energy $\left(E_{\mathrm{F}}\right)$ for both systems is 13.68 $\mathrm{eV}$ and lies at the edge of the conduction band indicating that both systems are n-type semiconductor which in agreement with the experimental result [22]. In order to obtain an accurate band gap value, we then rerun the NSCF calculation by choosing the "occupations = fixed" to treat the system as an insulator.

In Fig. 2, the SO interaction splits the band structure and gives a slightly difference in some regions especially at the $\mathrm{W}$ point. This situation, of course, affects the electronic and thermal properties of the material that can be seen in Fig. 3 and 4. The calculated (in-direct) band gap for both system is around $0.34 \mathrm{eV}$ which is very closed with the experimental result of $0.32 \mathrm{eV}$ [22].

\section{B. Electrical and Thermal Conductivity}

Fig. 3 (a) shows the temperature-dependent electrical conductivity per relaxation time $\tau$ of FeVSb system. We approximate that the value of $\tau$ is taken to be a constant, and its typical value around $10^{-14}-10^{-15}$ seconds for semiconductor materials.

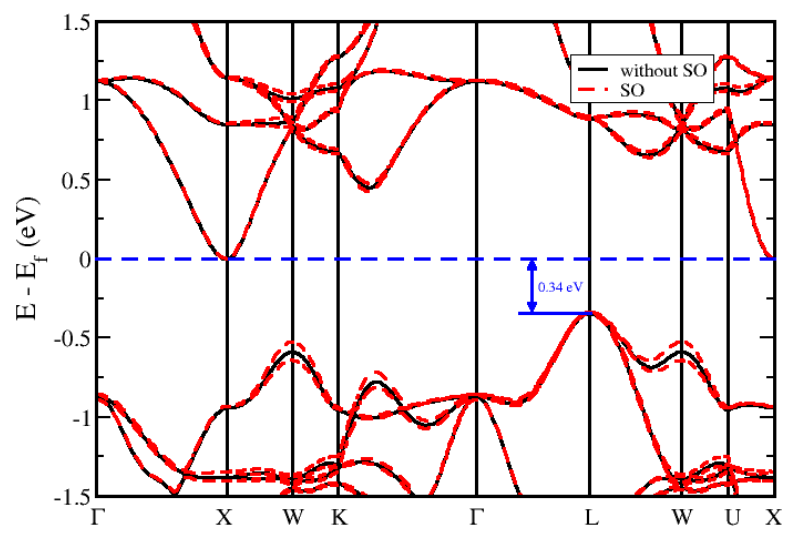

FIG. 2: Band structure of FeVSb system with (red line) and without (black line) spin-orbit interaction with in-direct band gap.
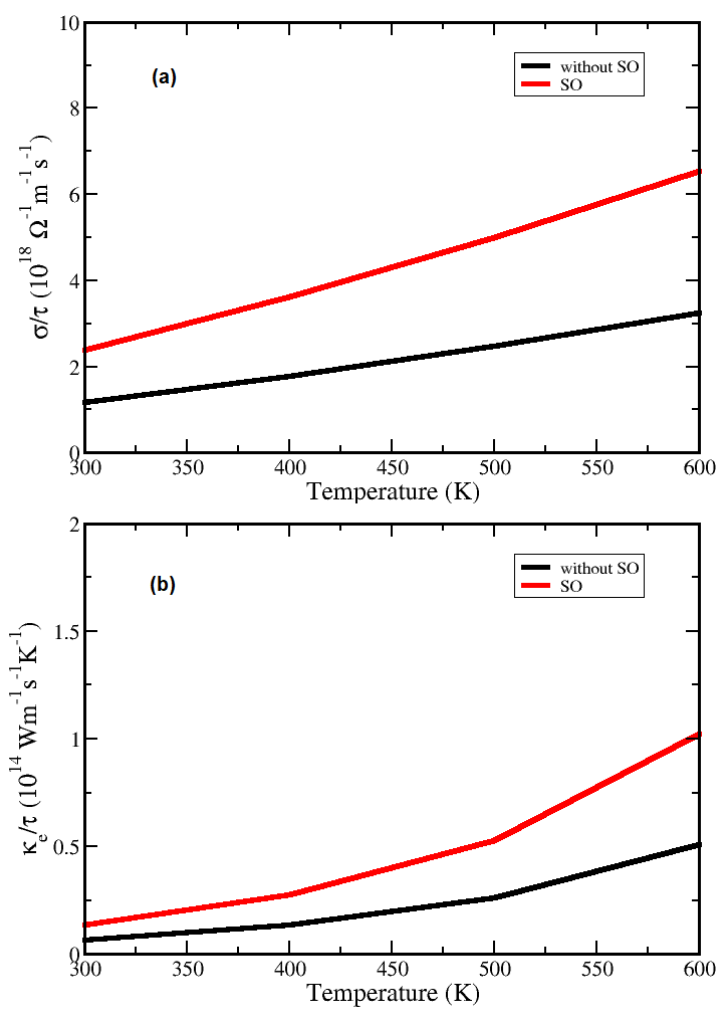

FIG. 3: The temperature dependent of (a) electrical conductivity $(\kappa)$ per relaxation time $(\sigma)$ and (b) electron thermal conductivity $\left(\kappa_{\mathrm{e}}\right)$ per relaxation time $(\tau)$ of the $\mathrm{FeVSb}$.

In Fig. 3 (a), the value of $\sigma / \tau$ for both systems is increased as the temperature is increased. The $\sigma / \tau$ value of the SO system is increased sharper than the wo-SO system due to the split bands that allow more electron to have higher energy than the wo-SO system. This situation will allow more electron to jump from the valance band into the conduction band. Our results show that both systems have good electrical conductivity values even for high-temperature regimes.

Fig. 3 (b) shows that the electron thermal conductivity 
(ETC) is increased as the temperature is increased. Although the $\sigma$ values of the SO system are higher than the wo-SO system, the $\kappa_{\mathrm{e}}$ values of the SO system are higher than the wo-SO system, which implies that the SO interaction does not only affect the $\sigma$ but also $\kappa_{\mathrm{e}}$, and give higher values of $\kappa_{\mathrm{e}}$ that will lower the figure of merit values as shown in Fig. 5. However, as we know that the real $\kappa$ is composed of two contributions which are the electron part and phonon part $\left(\kappa_{\mathrm{e}}=\kappa_{\mathrm{e}}+\right.$ $\left.\kappa_{\mathrm{ph}}\right)$. Experimentally, the lattice thermal conductivity can be minimized by using such as atomic substitution [14, 15], void filling method [13], and nano-sized the structure [12, 15].

\section{Seebeck Coefficient and Figure of Merit}

Fig. 4 shows the $S$ valus of FeVSb in the temperature range of $300-600 \mathrm{~K}$. As we see ini Fig. 4, the $S$ trends are quite shifted in the temperature range of $300-500 \mathrm{~K}$. The negative sign of the $S$ values indicates that electron is the major charge carrier for both systems. Our calculations give a good result to show that $\mathrm{FeVSb}$ is an n-type thermoelectric material even for high-temperature regime. Our results are also in agreement with the experimental report that give the Seebeck coefficient value of around $-100 \mu \mathrm{V} / \mathrm{K}$ in the temperature range of 300 - 600 K [23]. Despite our results have different Seebeck coefficient values, we have been successful to capture the main features of the FeVSb as n-type thermoelectric material signed by the negative Seebeck coefficient values.

From the Fig. 5, the figure of merit (ZT) values of FeVSb show that there are slightly shifted value for both systems similar to the Seebeck coefficient data in the temperature range of $300-600 \mathrm{~K}$. Both systems give a high ZT value even at $600 \mathrm{~K}$. Although the ZT values are still overestimated because we do not take into account the thermal conductivity contributed by the phonon. However, our calculation are qualitatively good to give the information about the main trend of the $\mathrm{ZT}$ values of $\mathrm{FeVSb}$ as a good candidate for the n-type thermoelectric materials. Vandu et al. reported that the ZT value of the HH TiNiSn system doped with $\mathrm{Zr}$ and $\mathrm{Hf}$ can achieve the value around 0.8 - 1.0 [24]. Therefore, there is a high possible chance to increase the $\mathrm{ZT}$ value of FeVSb such as by doping the system using other elements.

\section{SUMMARY}

We have done the first-principles density functional theory calculation to study the effect of spin-orbit interaction on the thermal properties of half-Heusler alloy FeVSb. The spin-orbit interaction gives the effect on the electronic and thermal properties of FeVSb. The energy gap of both systems is around $0.34 \mathrm{eV}$ which is very closed with the experimental report. The figure of merit values of FeVSb system with spin-orbit interaction are slightly lower than the FeVSb system without spin-orbit interaction due to its high $\kappa_{\mathrm{e}} / \tau$ values. The Seebeck coefficient and figure of merit (electron part) of both systems in the room temperature are around -242 $\mu \mathrm{V} / \mathrm{K}$ and 3.2, respectively. Both systems still give a high

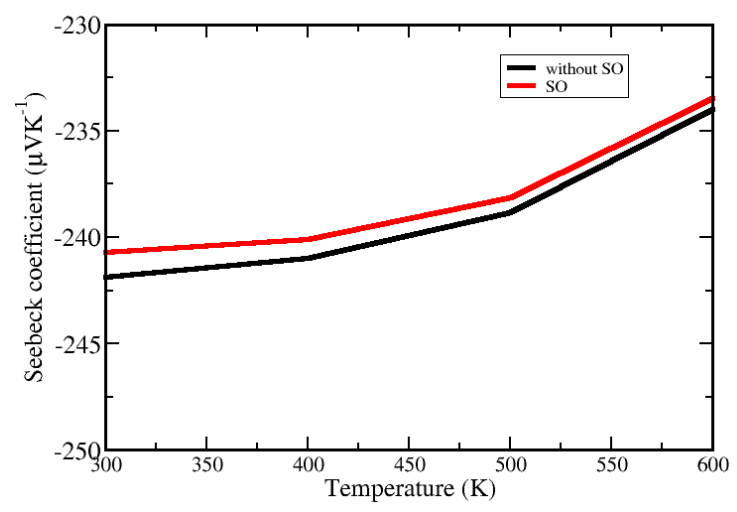

FIG. 4: Seebeck coefficient of FeVSb. The SO interaction gives higher Seebeck coefficient value than wo-SO system.

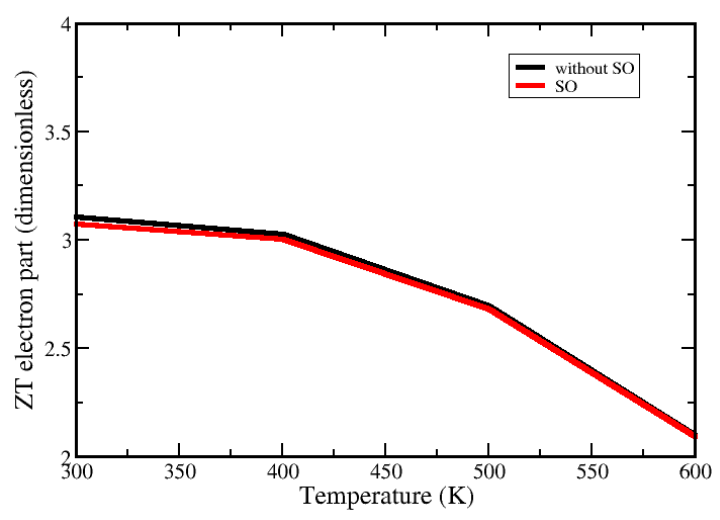

FIG. 5: Figure of merit (electron part) of FeVSb.

Seebeck coefficient and figure of merit (electron part) values in $600 \mathrm{~K}$ for around $-234 \mu \mathrm{V} / \mathrm{K}$ and 2.2 , respectively. Despite the thermal conductivity contributed by phonon is not taken into account, our result may not yet give a nearly realistic prediction if we compare our results with the real material. However, our results have been successfully captured the main trend of both the Seebeck coefficient and figure of merit of $\mathrm{FeVSb}$ as the n-type thermoelectric material signatured by the negative sign of their Seebeck coefficient values.

\section{Acknowledgment}

This research was supported by HPC facility of Indonesian Institute of Sciences (LIPI).
[1] F. Heusler and Verh, "Ueber magnetische Manganlegierungen," $D P G$, vol. 5, p. 220, 1903.
[2] T. Graf, C. Felser, and S. S. P. Parkin, "Simple rules for the 
understanding of Heusler compounds," Progress in Solid State Chemistry, vol. 39, no. 1, pp. 150, 2011.

[3] S. Skaftouros, K. Özdog̃an, E. Şaşog̃lu, and I. Galanakis, "Generalized Slater-Pauling rule for the inverse Heusler compounds," Physical Review B, vol. 87, no. 2, p. 024420, Jan. 2013.

[4] L. Wollmann, A. K. Nayak, S. S. P. Parkin, and C. Felser, "Heusler 4.0: Tunable Materials," Annual Review of Materials Research, vol. 47, no. 1, pp. 247270, Jul. 2017.

[5] T. Graf, C. Felser, and S. S. P. Parkin, "Heusler compounds: Applications in spintronics," in Handbook of Spintronics, Springer Netherlands, 2015, pp. 335364.

[6] C. Felser, L. Wollmann, S. Chadov, G. H. Fecher, and S. S. P. Parkin, "Basics and prospective of magnetic Heusler compounds," APL Materials, vol. 3, no. 4, 2015.

[7] V. Srivastava and K. P. Bhatti, "Ferromagnetic shape memory Heusler alloys," in Solid State Phenomena, vol. 189, Trans Tech Publications Ltd, 2012, pp. 189208.

[8] T. Klimczuk et al., "Superconductivity in the Heusler family of intermetallics," Physical Review B - Condensed Matter and Materials Physics, vol. 85, no. 17, May 2012.

[9] T. M. Tritt, "Thermoelectric Phenomena, Materials, and Applications," Annual Review of Materials Research, vol. 41, no. 1, pp. 433 448, Aug. 2011.

[10] L. Huang, Q. Zhang, B. Yuan, X. Lai, X. Yan, and Z. Ren, "Recent progress in half-Heusler thermoelectric materials," Materials Research Bulletin, vol. 76. Elsevier Ltd, pp. 107112, 01Apr-2016.

[11] X. Yan et al., "Enhanced thermoelectric figure of merit of ptype half-heuslers," Nano Letters, vol. 11, no. 2, pp. 556560, Feb. 2011.

[12] M. S. Dresselhaus et al., "New directions for low-dimensional thermoelectric materials," Advanced Materials, vol. 19, no. 8, pp. 10431053, 2007.

[13] G. S. Nolas, G. A. Slack, D. T. Morelli, T. M. Tritt, and A. C. Ehrlich, "The effect of rare-earth filling on the lattice thermal conductivity of skutterudites," Journal of Applied Physics, vol. 79, no. 8, pp. 40024008, 1996.
[14] Y. Kimura, H. Ueno, and Y. Mishima, "Thermoelectric properties of directionally solidified half-heusler (M 0.5a,M 0.5b )NiSn (M a, M b = Hf, Zr, Ti) alloys," in Journal of Electronic Materials, 2009, vol. 38, no. 7, pp. 934939.

[15] L. Huang, Q. Zhang, B. Yuan, X. Lai, X. Yan, and Z. Ren, "Recent progress in half-Heusler thermoelectric materials," Materials Research Bulletin, vol. 76, pp. 107112, 2016.

[16] S. Minami, F. Ishii, Y. P. Mizuta, and M. Saito, "First-principles study on thermoelectric properties of half-Heusler compounds CoMSb (M = Sc, Ti, V, Cr, and Mn)," Applied Physics Letters, vol. 113, no. 3, Jul. 2018.

[17] P. Giannozzi et al., "QUANTUM ESPRESSO: a modular and open- source software project for quantum simulations of materials," Journal of Physics: Condensed Matter, vol. 21, no. 39, p. 395502, Sep. 2009.

[18] P. Giannozzi et al., "Quantum ESPRESSO toward the exascale," Journal of Chemical Physics, vol. 152, no. 15, p. 154105 , Apr. 2020.

[19] G. K. H. Madsen and D. J. Singh, "BoltzTraP. A code for calculating band- structure dependent quantities," Computer Physics Communications, vol. 175, no. 1, pp. 6771, Jul. 2006.

[20] E. Gabriel et al., "Open MPI: Goals, Concept, and Design of a Next Generation MPI Implementation."

[21] A. Dal Corso, "Pseudopotentials periodic table: From $\mathrm{H}$ to Pu," Computational Materials Science, vol. 95, pp. 337350, Dec. 2014.

[22] R. Hasan and S. C. Ur, "Synthesis of Tin-Doped FeVSb HalfHeusler System by Mechanical Alloying and Evaluation of Thermoelectric Performance," Transactions on Electrical and Electronic Materials, vol. 19, no. 2, pp. 106111, Apr. 2018.

[23] A. El-Khouly et al., "Transport and thermoelectric properties of Hf-doped FeVSb half-Heusler alloys," Journal of Alloys and Compounds, vol. 820, p. 153413, Apr. 2020.

[24] N. Van Du et al., "X-site aliovalent substitution decoupled charge and phonon transports in XYZ half-Heusler thermoelectrics," Acta Materialia, vol. 166, pp. 650657, Mar. 2019. 